\title{
Large-scale optical-field measurements with geometric fibre constructs
}

\author{
AYMAN F. ABOURADDY ${ }^{1}$, OFER SHAPIRA ${ }^{1,2}$, MEHMET BAYINDIR ${ }^{1 *}$, JERIMY ARNOLD², FABIEN SORIN ${ }^{3}$, \\ DURSEN S. HINCZEWSKI ${ }^{3}$, JOHN D. JOANNOPOULOS ${ }^{1,4}$ AND YOEL FINK ${ }^{1,3 \dagger}$
}

\author{
${ }^{1}$ Research Laboratory of Electronics, Massachusetts Institute of Technology, Cambridge, Massachusetts 02139, USA \\ ${ }^{2}$ Department of Electrical Engineering and Computer Science, Massachusetts Institute of Technology, Cambridge, Massachusetts 02139, USA \\ ${ }^{3}$ Department of Materials Science and Engineering, Massachusetts Institute of Technology, Cambridge, Massachusetts 02139, USA \\ ${ }^{4}$ Department of Physics, Massachusetts Institute of Technology, Cambridge, Massachusetts 02139, USA \\ *Present address: Department of Physics, Bilkent University, Ankara 06800, Turkey \\ †e-mail: yoel@mit.edu
}

0 ptical fields are measured using sequential arrangements of optical components such as lenses, filters, and beam splitters in conjunction with planar arrays of point detectors placed on a common axis ${ }^{1}$. All such systems are constrained in terms of size, weight, durability and field of view. Here a new, geometric approach to optical-field measurements is presented that lifts some of the aforementioned limitations and, moreover, enables access to optical information on unprecedented length and volume scales. Tough polymeric photodetecting fibres drawn from a preform ${ }^{2}$ are woven into light-weight, low-optical-density, two- and three-dimensional constructs that measure the amplitude and phase of an electromagnetic field on very large areas. First, a threedimensional spherical construct is used to measure the direction of illumination over $4 \pi$ steradians. Second, an intensity distribution is measured by a planar array using a tomographic algorithm. Finally, both the amplitude and phase of an optical wave front are acquired with a dual-plane construct. Hence, the problem of optical-field measurement is transformed from one involving the choice and placement of lenses and detector arrays to that of designing geometrical constructions of polymeric, light-sensitive fibres.

The arrays are constructed of photodetecting fibres produced by a recently developed fabrication process that enables the incorporation of insulators, conductors, and semiconductors in mesostructured fibres combining both optical and electrical functionalities ${ }^{2}$. Specifically, the fibres we make use of in this paper are constructed of a photoconductive glass core which is in intimate contact with metal electrodes that run along the length of the fibre and is surrounded by a protective, transparent polymer cladding, as shown in Fig. 1a (see the Methods section). The resulting fibres are arbitrarily long, light-weight, flexible, one-dimensional (1D) light-sensitive elements that produce an electrical signal in the form of a change in current in an external circuit when light impinges on their external surface, as illustrated in Fig. 1b,c (refs 2,3). It is interesting to note that each fibre is an omnidirectional detector in the sense that it detects an incident light beam regardless of the relative angle between the fibre and the beam. This is in sharp contrast with traditional optical detectors that can detect light incident from (at best) one-half of ambient space. Nevertheless, a single fibre is not able to determine the angle of incidence, a task that is accomplished through arranging a multiplicity of fibres in an array.

The flexibility of these fibres allows us to design a directional light-detection arrangement through the construction of an optical array or 'web' with a novel topology: that of a closed-surface sphere (see the Methods section). In the limit of geometric optics, any beam of light (with a cross-section larger than the separation between the fibres) incident on the array must intersect the array again on exit (except for tangential beams). Such an array, as shown in Fig. 2a, provides directional detection over a full $4 \pi$ solid angle. Figure $2 \mathrm{a}$ highlights several distinctive features of our approach. The fibres are mechanically tough, yet very flexible, such that the array is near self-supporting from a mechanical standpoint. Furthermore, the array is sufficiently sparse, and the fibres are sufficiently thin that the array is rendered near transparent: the incident beam is only partially absorbed on entrance, propagates to the exit point where it is partially absorbed again, traversing the structure with little overall impediment. The distribution of the detected electrical signals from the fibres across the sphere is shown in Fig. 2b for three different incident light beams that are shown in Fig. 2c, clearly demonstrating the directional lightdetection capability of this topology.

We now proceed to demonstrate a more sophisticated task, namely the detection of an arbitrary optical intensity distribution using a planar fibre web. An important observation brings to the fore which body of theoretical work is of relevance to this problem. As each fibre detects the incident intensity distribution along its whole length, the intercepted power (and, consequently, the electrical signal produced) is therefore a line integral of the intensity distribution along the fibre. A fibre of length $\Lambda$ and photodetecting diameter $\Delta(\Delta \ll \Lambda)$ placed along the line $x \cos \theta+y \sin \theta=t_{1}$ in an optical field with a 2D intensity distribution $I(x, y)$, as illustrated in Fig. 3a, generates a photocurrent that is proportional to the 

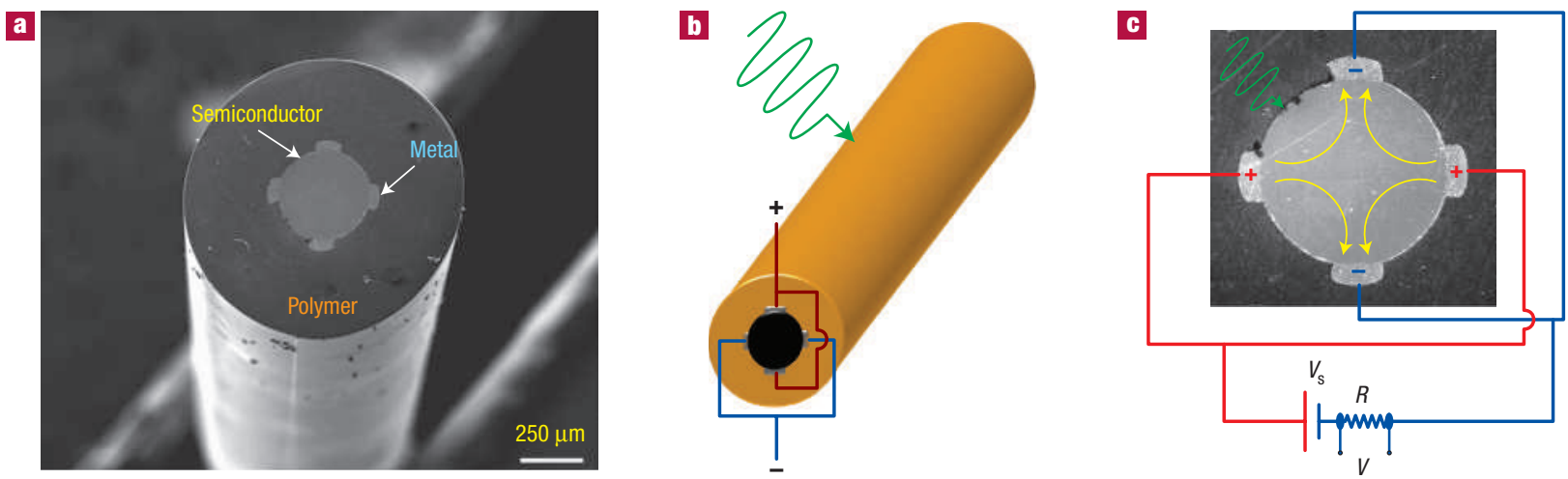

Figure 1 Photodetecting fibres. a, Scanning electron micrograph of a photodetecting fibre cross-section. b, A schematic diagram of a 1D fibre photodetector with light (green arrow) incident on the external surface. c, The fibre electrodes are connected to an external circuit and the associated electric field lines (yellow arrows) are shown.

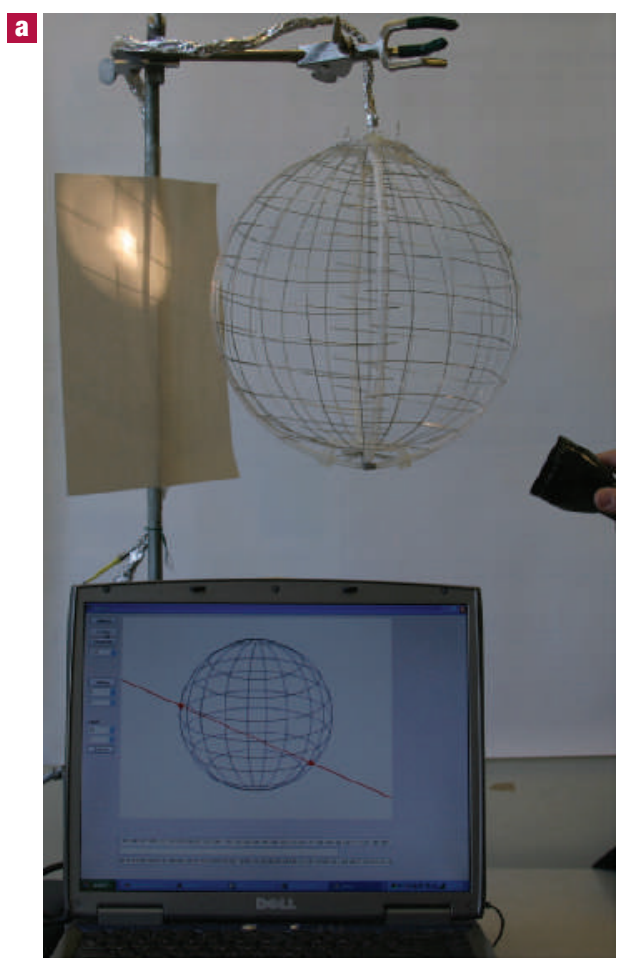

b
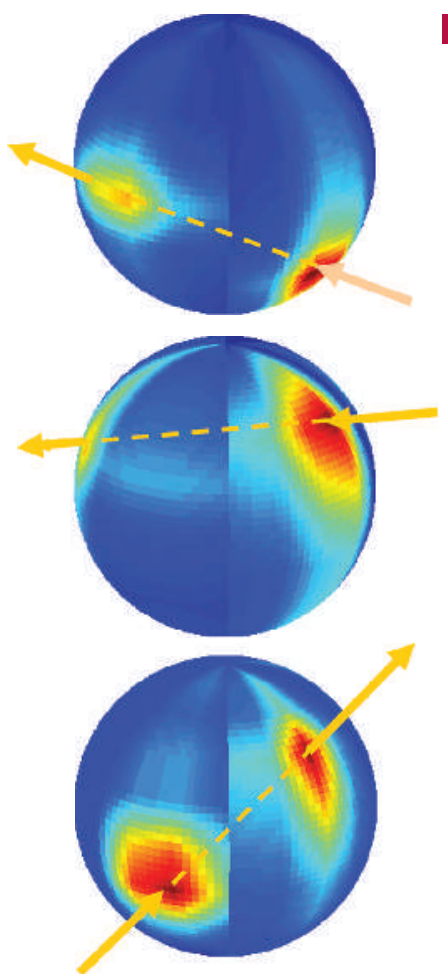

$\mathbf{G}$
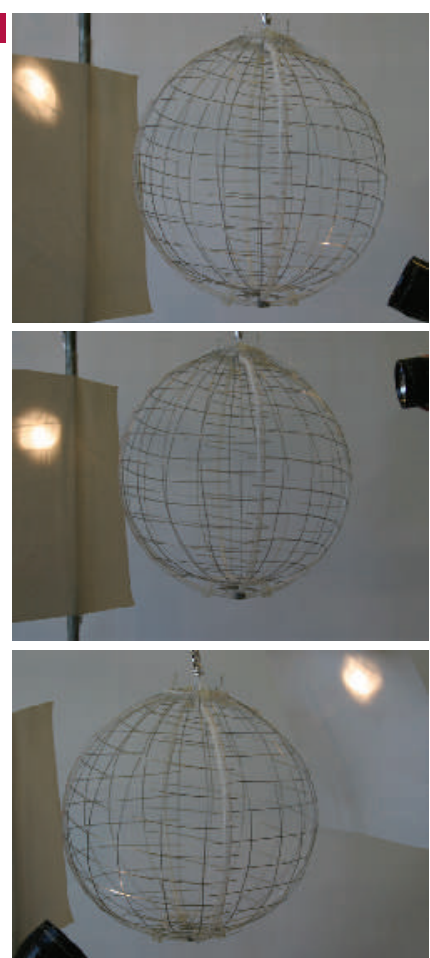

Figure 2 Omnidirectional light detection. a, A closed spherical fibre web is an omnidirectional photodetector which detects the direction of the beam throughout a solid angle of $4 \pi$. The spherical web is sufficiently transparent to see through and for a beam of light to traverse unimpeded. $\mathbf{b}$, The distribution of the electrical signals detected by the fibres for a light beam incident in three different directions. The arrows indicate the direction of the beams, and the dashed portion of each arrow corresponds to the beam's path inside the sphere. c. Photographs of the three beam trajectories that resulted in the signal distributions shown in $\mathbf{b}$.

intercepted optical power $P_{\theta}\left(t_{1}\right)$, given by

$$
\begin{aligned}
P_{\theta}\left(t_{1}\right) & =\iint_{\text {fibre area }} \mathrm{d} x \mathrm{~d} y I(x, y) \\
& \approx \Delta \iint \mathrm{d} x \mathrm{~d} y I(x, y) \delta\left(x \cos \theta+y \sin \theta-t_{1}\right),
\end{aligned}
$$

where $t_{1}$ is the distance from the fibre to the origin and $\delta$ is the Dirac-delta function. The approximation used in this equation is to consider the incident intensity constant across the photoconductive core thickness $\Delta$ (but not along the length $\Lambda$ ).

Consequently, the measurements carried out by a set of parallel photodetecting fibres form a 'parallel projection' of the incident intensity distribution, a term used in the literature on computerized axial tomography $(\mathrm{CAT})^{4}$. In that context it refers to the measurements carried out by a linear array of point detectors placed on one side of a 2D object of interest, when a linear array of point sources (for example, X-rays) is placed on the opposite side of the object. In our case, each fibre records the line integral 

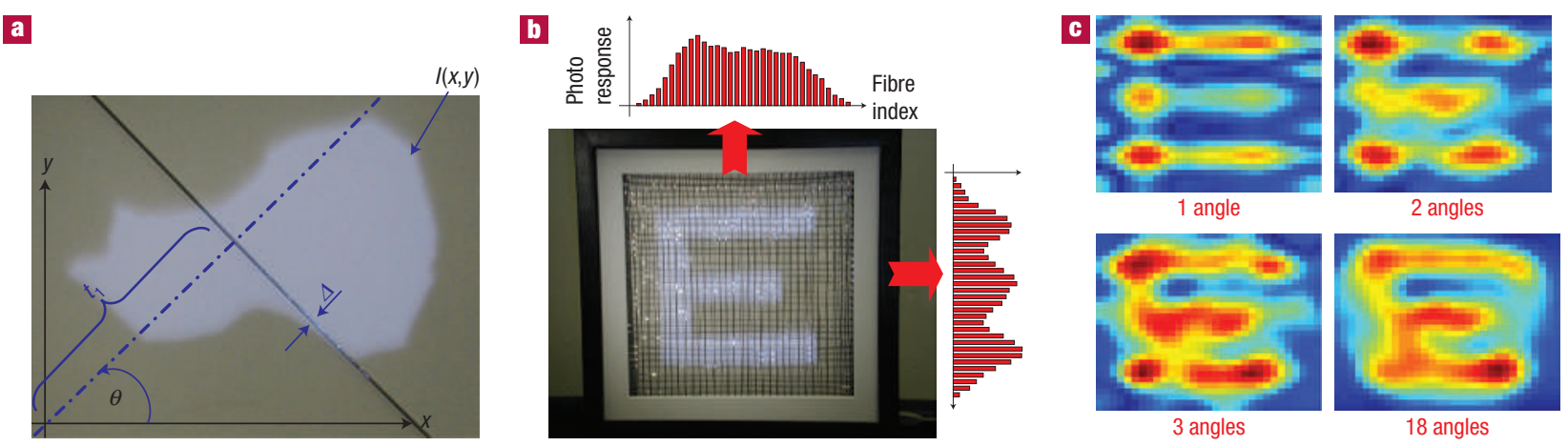

Figure 3 Reconstructing an arbitrary optical intensity distribution with a planar fibre web. a, A photodetecting fibre of thickness $\Delta$ detects the line integral of the arbitrary incident optical intensity distribution, $I(x, y)$. $\mathbf{b}$, An image of the letter ' $E$ ' is projected onto a $32 \times 32$ fibre web of dimensions $24 \times 24 \mathrm{~cm}^{2}$. The detected electrical signals from the web rows and columns (constituting 2 orthogonal projections) are also shown. The image of the letter 'E' seen here is formed on a white sheet placed behind the transparent web. c, Reconstructions of the incident intensity distribution, obtained using the backprojection algorithm, are shown with increasing number of projections. The projections are obtained by rotating the object transparency. 1 angle: $0^{\circ} ; 2$ angles: $0^{\circ}, 45^{\circ} ; 3$ angles: $0^{\circ}, 30^{\circ}, 60^{\circ}$; and 18 angles: $0^{\circ}$ to $85^{\circ}$ in $5^{\circ}$ steps. Note that each angle corresponds to two projections obtained by the fibre rows and columns independently.

of the intensity distribution of the optical field along its length. An example of a parallel projection produced by a fibre web is shown in Fig. 3b, where a $32 \times 32$ fibre web (of dimensions $24 \times 24 \mathrm{~cm}^{2}$ ) intercepts an image of a letter ' $E$ ' and two orthogonal projections obtained by the rows and columns of the web are displayed. The image is produced by a white-light lamp (Xe-Hg) illuminating a transparency with dimensions $14 \times 14 \mathrm{~mm}^{2}$ placed at a distance of $1.2 \mathrm{~m}$ from the web. No lens is needed to form an image of the object in this case because of the large dimensions used (relative to the wavelength of light), highlighting a unique advantage of a detector array with such a large area.

The description of these two apparently different arrangements (CAT and our fibre webs) are, surprisingly, mathematically isomorphic, allowing us to import the theoretical foundations of CAT for use in the problem at hand. In particular, we use the backprojection algorithm (Radon transform) ${ }^{4}$, commonly used in CAT, to reconstruct an estimate of the impinging optical intensity distribution from a set of rotated projections. In the case of fibre webs there are several strategies to obtain these projections: (i) rotating the fibre web; (ii) using adjacent or interleaved fibre webs rotated with respect to each other; or (iii) rotating the object that is being imaged. In Fig. $3 c$, we show the image reconstructions with increasing number of projections recorded by rotating the object. Increasing the number of acquired projections improves the fidelity of the reconstructed image to the incident intensity distribution.

Although a single planar fibre web can detect only the intensity of an incident optical field, two such webs can be used to reconstruct both the field amplitude and phase, which constitute a complete representation of a scalar optical field in the waveoptics regime. We now proceed to demonstrate this capability by using two planar fibre webs to reconstruct an object from intensity measurements of the diffracted field by using the phase-retrieval algorithm ${ }^{5-7}$. In its simplest form, this algorithm iteratively obtains the phase of a wave front if the amplitude is known in two different planes (see the Methods section). The object used was a letter ' $E$ ' with an overall size of $750 \times 750 \mu \mathrm{m}^{2}$ (with feature sizes of $150 \mu \mathrm{m}$; the object was a chrome mask on a glass substrate) illuminated with laser light at a wavelength of $830 \mathrm{~nm}$ (see the Methods section and Fig. 4a). The 2D intensity distribution data obtained by a fibre web in two different planes in the far-field of the object is shown in Fig. 4b. We implemented the phase-retrieval algorithm on the two obtained intensity distributions to reconstruct the complex field at an arbitrary plane. To verify the fidelity of the field estimation, we back propagate the field computationally to planes receding from the first web towards the object. A set of these computed distributions is shown in Fig. 4b. The estimates are blurred until we approach the location of the object whereupon a clear image is formed. The performance of this approach is compared with a theoretical model in which we calculated the diffracted fields of the letter ' $E$ ' modulated by a gaussian beam (representing the illuminating beam profile of the laser) at the two measurement planes. The resulting amplitude distributions were truncated to the array size and discretized on $32 \times 32$ grids shown in Fig. $4 c$. We then applied the phase-retrieval algorithm to these two theoretical images to reconstruct the object, and good agreement between experiment and theory is observed (compare Fig. $4 \mathrm{~b}$ and c).

This approach may eventually become useful in optical imaging when a larger number of fibres are included in the web to form images of objects with more detail. Note that this system has an infinite depth of focus, that is, an image of the object is formed regardless of the distance of the object from the webs, provided that the diffracted field at the locations of the two webs is intercepted. Furthermore, the image reproduces the object with its physical dimensions and also determines its physical distance from the webs. In principle, by virtue of obtaining a complete description (both the amplitude and phase) of the electromagnetic field, this approach may be used to image $3 \mathrm{D}$ objects that are translucent enough such that excessive occlusion does not occur. A distinct approach relies on the use of a single dense array with a higher fill factor that acquires a single intensity measurement ${ }^{8}$. This procedure requires that an over-sampling condition be satisfied: the sampling of the diffracted field must be dense enough to enable the reconstruction of the object autocorrelation function ${ }^{9,10}$.

Finally, we comment on the physical dimensions of such arrays. A 40-mm-diameter, 30-cm-long preform can be drawn down to a 48 -km-long fibre with a $100-\mu \mathrm{m}$ diameter. A low-optical-density $15 \times 15 \mathrm{~m}^{2}$ array can then be formed with $1-\mathrm{cm}$ spacing providing $2,250,000$ detection points. The total photosensitive area of this array is approximately $4.5 \mathrm{~m}^{2}$. With the average density of the fibre materials being about $2 \mathrm{~g} \mathrm{~cm}^{-3}$, the overall weight of this array is approximately $700 \mathrm{~g}$, which is negligible when compared with the weight of any traditional optical component of comparable size. This in turn enables the assembly of very large optical detection 

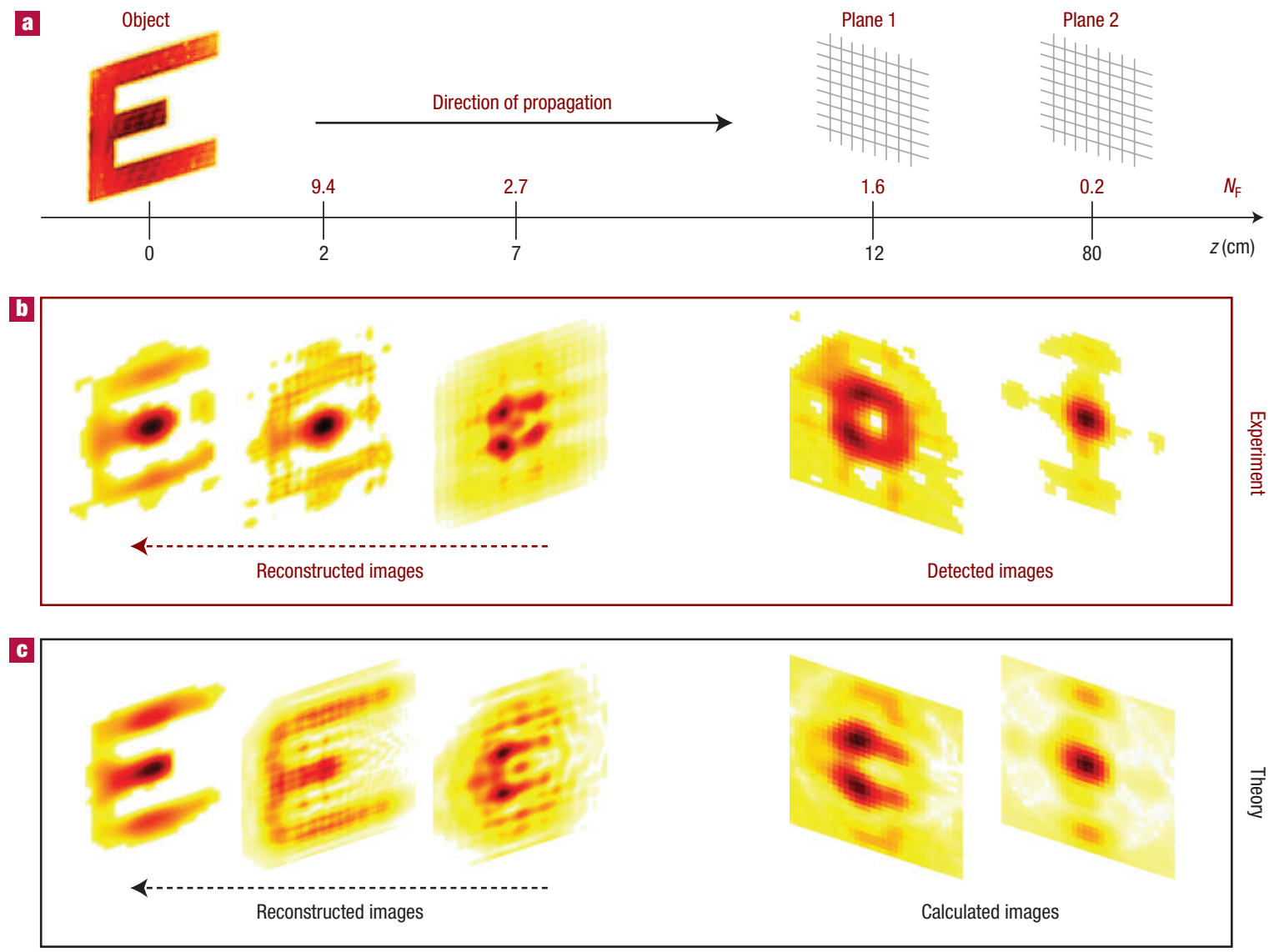

Figure 4 Non-interferometric lensless imaging using two fibre webs. a, An object (letter 'E') is illuminated with a laser beam (the image of the object is captured with a Vidicon camera) and the propagating diffracted field amplitude is obtained at two different locations using $32 \times 32$ planar fibre webs. The Fresnel number ${ }^{1} N_{\mathrm{F}}$ is also given. b. Two intensity distributions are measured. The phase-retrieval algorithm is used to obtain back-propagated images in the direction receding from the webs towards the object (the object reconstruction is at $0 \mathrm{~cm}$, shown on the left). The reconstructed images are blurred, but an image is obtained at the location of the object. c, The second row shows the results of theoretical reconstructions with the phase-retrieval algorithm when using the calculated diffraction patterns that were obtained from the measured illuminated object distribution.

systems that could sample electromagnetic fields on unprecedented length scales.

The fibre arrays discussed in this paper may acquire intensity distributions, identify the direction of propagation, and even extract the amplitude and phase of the optical field, and they can also be designed to access additional electromagnetic field attributes, such as wavelength ${ }^{2}$ and possibly polarization, by modifying the geometry of the fibre. In addition, changing the composition of the semiconducting glass will enable sensitivity to other wavelength regions in the electromagnetic spectrum and even to other physical quantities, such as acoustic waves, temperature ${ }^{11}$, or chemical contaminants. Webs constructed of such fibres, using the same principles outlined above, will yield 'images' in these parameter spaces, and can thus be said to see, hear, sense heat, and smell.

It is important to note that the optical imaging systems carrying out the tasks all consist solely of these fibres with no other optical components or detectors required. Light traverses an array of such fibres essentially unhindered and may be considered to be transparent. In his 1897 novel, 'The Invisible Man', H. G. Wells explores the consequences and possibilities of a human rendered invisible to others. Understandably, the novel roused many objections, one being that a truly invisible man would necessarily be blind. Our approach provides an intriguing new insight into this problem, as the fibre arrays could be made to be nearly transparent yet possess simple 'vision' capabilities; such a system seems to be the closest realization to Wells' fantasy.

\section{METHODS}

\section{FIBRE PREPARATION}

The photoconductive glass used was the amorphous chalcogenide semiconductor $\mathrm{As}_{40} \mathrm{Se}_{49} \mathrm{Te}_{11} \mathrm{Sn}_{5}$. This tin-doped AST chalcogenide glass has a transparency window between 1.2 and $16 \mu \mathrm{m}$, and is prepared from high-purity (5-6N) As, Se, Te, and Sn elements using sealed-ampoule melt-quenching techniques. The materials were weighed and placed into a quartz tube under a nitrogen atmosphere. The tube was heated to $330^{\circ} \mathrm{C}$ for $1 \mathrm{~h}$ at a rate of $1{ }^{\circ} \mathrm{C} \mathrm{min}{ }^{-1}$ under vacuum to remove surface oxide, $\mathrm{As}_{2} \mathrm{O}_{3}$, and cooled to room temperature at the same rate. The ampoule was formed by sealing the tube under vacuum $\left(\sim 10^{-5}\right.$ torr). It was then heated to $800^{\circ} \mathrm{C}$ at a rate of $2{ }^{\circ} \mathrm{C} \mathrm{min}^{-1}$ in a rocking furnace, while being held vertical, for $24 \mathrm{~h}$ and then rocked for $6 \mathrm{~h}$ to increase mixing and homogenization. The glass liquid was cooled to $600^{\circ} \mathrm{C}$ in the furnace, and then quenched in water. Subsequently, it was annealed for $30 \mathrm{~min}$ near the glass-transition temperature, $T_{\mathrm{g}}=180^{\circ} \mathrm{C}$, before being cooled gradually to room temperature.

The hundreds of metres of fibre were obtained from a macroscopic cylindrical preform, 33- $\mathrm{mm}$ in diameter and $25-\mathrm{cm}$ in length, which consists of the Sn-doped AST glass core ${ }^{2}$ contacted by four alloy $(\mathrm{Sn}-5 \% \mathrm{Ag})$ conduits that 
are encapsulated in a protective polymer (polysulphone) cladding, which is transparent in the visible and near-infrared wavelengths. The preform was consolidated for $60 \mathrm{~min}$ at $230^{\circ} \mathrm{C}$ under vacuum in a three-zone horizontal tube furnace while rotating the preform around its axis. Subsequently, the preform was drawn in a three-zone vertical tube furnace with the top-zone temperature between 165 and $200^{\circ} \mathrm{C}$ and the middle-zone temperature $270^{\circ} \mathrm{C}$.

\section{CONSTRUCTION OF FIBRE WEBS}

The distribution of the photodetecting fibres arranged on the spherical array has the familiar geometry of longitudes and latitudes on a globe, except that each latitude consists of two fibres each forming half a circle. There are 20 fibres, of equal length, equally spaced on the longitudes. The 22 latitudinal fibres have different lengths (from the equator to the two poles). Each planar fibre web is constructed of 64 photodetecting fibres arranged on a $32 \times 32$ square grid with clear dimensions of $24 \times 24 \mathrm{~cm}^{2}$. The frame used to hold these fibres is a multilayer printed circuit board with accommodation for electrical connection to the fibre and mechanical support. Two electrodes running along the core of the fibres are electrically contacted, using silver paint and two wires, to the external circuit. The 'dark' impedance of each fibre is approximately $140 \mathrm{M} \Omega$. The circuitry of the planar and spherical webs is similar. Each fibre is connected to a high-input-impedance operational amplifier (precision low-power JFET amplifier, AD8625) in a current-amplifier configuration. The amplified signals are multiplexed (using high-speed complementary metal-oxide semiconductor 16-channel multiplexers, CD74HCT4067), and then digitized by an 8-bit multichannel analog-to-digital converter on a Microchip microcontroller (PIC16C745) before being converted to a universal serial bus (USB) format. All of these electronic components are located on the printed circuit board used as a frame. A USB cable then connects the fibre web to a personal computer that activates the web and collects the detected signals. No power supply is needed for the fibre webs, and power to operate the circuitry is derived from the USB ports on the personal computer.

\section{PHASE-RETRIEVALALGORITHM}

We define an error metric $\Theta=\int \mathrm{d} \mathbf{r} \sum_{n=1,2}\left(\left|G_{n}(\mathbf{r})\right|^{2}-I_{n}(\mathbf{r})\right)^{2}$ describing the functional distance between an estimate $\left|G_{n}\right|^{2}$ and a measured intensity distribution $I_{n}$ in both diffraction planes, $n=1,2$. The distributions in the two diffraction planes are related to the object distribution $g(x, y)$ through the Fresnel transform $\mathfrak{I}, G_{n}(u, v)=\Im\{g\}(u, v)$. The Nyquist frequency for such a system is $1 / L$, where $L$ is the size of $g$ (in one dimension) and the sampling rate corresponds to fibre spacing $\angle \lambda D / L$, where $\lambda$ is the wavelength and $D$ is the distance between the object and the array. The array size sets a cutoff for the spatial frequencies and therefore determines the sharpness of the reconstructed image. The phase-retrieval algorithm used requires the minimization of the metric $\Theta$ with respect to the set of $2 \mathrm{D}$ variables $\{g(n, m)\}$, where $n$ and $m$ are discretized spatial coordinates. The minimization is carried out iteratively by repeating the following steps. (i) Calculate $G_{n}$ in both planes using the previous estimate of $g$ (an initial guess for $g$ is needed in the first iteration). (ii) Calculate $\Theta$ and its partial derivatives with respect to $g(n, m)$. (iii) Using the gradient-search method, find the next estimate of $g$ (with smaller error). These steps are repeated until the change in the error metric is below a threshold limited by the system noise.

We comment on the extent of the disturbance to the optical field produced by absorption of a portion of the impinging optical power by the fibre array. Consider the general situation shown in Fig. 4 a, where an optical field $E_{1}(x, y)$ incident on the first array is perturbed by the first planar array before propagating to the plane of the second array where the field is $E_{2}(x, y)$. To investigate the limits placed on this procedure owing to diffraction caused by the fibres in the first array, assume that all of the fibres in the first array are identical, have an effective width $\Delta$ and are arranged on a uniform square grid with separations $d$. The incident field $E_{1}(x, y)$ is modulated by the $2 \mathrm{D}$ transfer function of the array $T_{2 \mathrm{D}}(x, y)$, such that the field directly after the array is $E_{1}(x, y) \cdot T_{2 \mathrm{D}}(x, y)$. The array transfer function is separable, $T_{2 \mathrm{D}}(x, y)=T_{1 \mathrm{D}}(x) \cdot T_{1 \mathrm{D}}(y)$, with $T_{1 \mathrm{D}}(x)=1-\operatorname{rect}(x / \Delta)$ $\otimes \sum_{n=-N}^{N} \delta(x-n d)$ where $\otimes$ stands for the convolution process; $\operatorname{rect}(x / \Delta)=1$ when $|x| \leq \Delta / 2$ and is 0 elsewhere; $2 N+1$ fibres are assumed along one direction. We assume that the first fibre array is large enough to capture most of the incident field $E_{1}(x, y)$. This allows us to let $N$ formally become infinite, and the Fourier transform of $T_{1 \mathrm{D}}$ becomes

$$
\operatorname{FT}\left\{T_{1 \mathrm{D}}\right\}(u) / 2 \pi=\delta(u)-\frac{\Delta}{d} \cdot \frac{\sin u \Delta / 2}{u \Delta / 2} \sum_{n=-\infty}^{\infty} \delta\left(u-2 \pi \frac{n}{d}\right),
$$

where $\delta(u)$ is the Dirac-delta function. Note that the zeroth-order component in the second term $(n=0)$ on the right-hand side of equation (1) is weighted by a factor of $\Delta / d$ with respect to the first term. After propagating a distance $d$ to the second array, the diffracted field $E_{2}(x, y)$ is given by the Fresnel integral of the modulated incident field, $E_{2}(u, v)=\Im\left\{E_{1} T_{2 \mathrm{D}}\right\}(u, v)=\Im\left\{E_{1}\right\}(u, v)$ $\otimes \operatorname{FT}\left\{T_{2 \mathrm{D}}\right\}(u, v)$ (we have ignored an unimportant overall phase term). Two conditions need to be met: (i) the size of the second array should be smaller than the separation between the delta functions in the second term on the right-hand side of equation (1), and (ii) $\Delta / d \ll 1$ (by using fibres of thickness much smaller than the fibre spacing) such that the contribution of the zeroth-order second term in equation (1) becomes negligible; $E_{2}(x, y)$ becomes approximately the Fresnel transform of $E_{1}$.

A gaussian beam, from a Ti:sapphire laser tuned to a wavelength of $830 \mathrm{~nm}$, is incident perpendicularly on an amplitude mask with the letter ' $E$ ', generating a Fresnel diffraction pattern $12 \mathrm{~cm}$ away from the mask with size of $\sim 1 \mathrm{~mm}$, and a Fraunhofer diffraction pattern $80 \mathrm{~cm}$ away with size of $\sim 6.5 \mathrm{~mm}$. In this proof-of-principle experiment, we magnified those patterns to match the array size. We implemented the above-described phase-retrieval algorithm using an optimization routine from MATLAB, and optimized the error function to obtain the reconstructed field at the plane of the mask. The iterative process converged after less than 20 iterations to an error of less than $1 \%$. An estimate of the amplitude and phase of the field in one plane can be used to propagate the field to any other plane, and a 'focused' image is observed at a distance that matches the location of the object. We compared these results with a theoretical model that numerically propagates the field from the object, using the object distribution modulated by the incident laser beam (as captured by a Vidicon camera) and taking into consideration the $32 \times 32$ discretization of the diffracted fields at the measurement planes. The reconstructed fields and calculated images are shown in Fig. $4 \mathrm{~b}$ and c, respectively.

\section{Received 2 December 2005; accepted 15 May 2006; published 25 June 2006.}

References

1. Saleh, B. E. A. \& Teich, M. C. Fundamentals of Photonics (Wiley, New York, 1991)

2. Bayindir, M. et al. Metal-insulator-semiconductor optoelectronic fibres. Nature 431, 826-829 (2004)

3. Bayindir, M. et al. Fibre photodetectors codrawn from conducting, semiconducting and insulating materials. Opt. Photon. News 15, 24 (2004).

4. Kak, A. C. \& Slaney, M. Principles of Computerized Tomographic Imaging (IEEE Press, New York, 1988).

5. Gerchberg, R. W. \& Saxton, W. O. A practical algorithm for the determination of phase from image and diffraction plane pictures. Optik 35, 237-246 (1972).

6. Saxton, W. O. Computer Techniques for Image Processing in Electron Microscopy (Academic, New York, 1978).

7. Fienup, J. R. Phase retrieval algorithms: a comparison. Appl. Opt. 21, 2758-2769 (1982).

8. Cederquist, J. N., Fienup, J. R., Marron, J. C. \& Paxman, R. G. Phase retrieval from experimental far-field speckle data. Opt. Lett. 13, 619-621 (1988).

9. Bates, R. H. T. Fourier phase problems are uniquely soluble in more then one dimension. I: Underlying theory. Optik 61, 247-262 (1982).

10. Miao, J., Sayre, D. \& Chapman, H. N. Phase retrieval from the magnitude of the Fourier transforms of nonperiodic objects. J. Opt. Soc. Am. A 15, 1662-1669 (1988).

11. Bayindir, M., Abouraddy, A. F., Arnold, J., Joannopoulos, J. D. \& Fink, Y. Thermal fibre grids for spatially resolved heat detection on large areas. Adv. Mater. 18, 845-849 (2006).

\section{Acknowledgements}

We thank N. Orf, Y. Migdal and E. E. Bayindir for technical assistance, and M. Qi for useful discussions. This work was supported by the ISN DAAD-19-02-D-0002, US DOE

DE-FG02-99ER45778 and DARPA/Christodoulou. This work was also supported in part by the MRSEC Program of the National Science Foundation under award number DMR 02-13282. Correspondence and requests for materials should be addressed to Y.F.

\section{Competing financial interests}

The authors declare that they have no competing financial interests.

Reprints and permission information is available online at http://npg.nature.com/reprintsandpermissions/ 